\title{
Erratum
}

\section{A Note to Paper "On the Stability of Cubic Mappings and Quartic Mappings in Random Normed Spaces"}

\author{
R. Saadati, ${ }^{1}$ S. M. Vaezpour, ${ }^{1}$ and Y. J. Cho ${ }^{2}$ \\ ${ }^{1}$ Department of Mathematics and Computer Science, Amirkabir University of Technology, \\ 424 Hafez Avenue, Tehran 15914, Iran \\ ${ }^{2}$ Department of Mathematics Education and the RINS, Gyeongsang National University, \\ Chinju 660-701, South Korea
}

Correspondence should be addressed to Y. J. Cho, yjcho@gnu.ac.kr

Received 16 February 2009; Accepted 21 April 2009

Recently, Baktash et al. (2008) proved the stability of the cubic functional equation $f(2 x+y)+f(2 x-$ $y)=2 f(x+y)+2 f(x-y)+12 f(x)$ and the quartic functional equation $f(2 x+y)+f(2 x-y)=$ $4 f(x+y)+4 f(x-y)+24 f(x)-6 f(y)$ in random normed spaces. In this note, we correct the proofs.

Copyright (C) 2009 R. Saadati et al. This is an open access article distributed under the Creative Commons Attribution License, which permits unrestricted use, distribution, and reproduction in any medium, provided the original work is properly cited.

\section{Introduction and Preliminaries}

If $\inf \{t>0: F(t)>a\} \leq \inf \{t>0: G(t)>a\}$, in general we cannot conclude that $F(t) \geq G(t)$. For example, let $F(t)=3 / 4, G(t)=t /(t+1)$ and $a=1 / 2$. We know that $\inf \{t>0: 3 / 4>$ $1 / 2\}=0 \leq \inf \{t>0: t /(t+1)>1 / 2\}=1$ but $F(4)=3 / 4<G(4)=4 / 5$. This example shows that in [1], inequalities (2.13) and (3.13) do not follow from inequalities (2.12) and (3.12).

The functional equation

$$
f(2 x+y)+f(2 x-y)=2 f(x+y)+2 f(x-y)+12 f(x)
$$

is said to be the cubic functional equation since the function $f(x)=c x^{3}$ is its solution. Every solution of the cubic functional equation is said to be a cubic mapping. The stability problem for the cubic functional equation was solved by Jun and Kim [2] and Lee [3] for mappings $f: X \rightarrow Y$, where $X$ is a real normed space and $Y$ is a Banach space. Later, a number of mathematicians have worked on the stability of some types of the cubic equation [4]. The functional equation

$$
f(2 x+y)+f(2 x-y)=4 f(x+y)+4 f(x-y)+24 f(x)-6 f(y)
$$


is said to be the quartic functional equation since the function $f(x)=c x^{4}$ is its solution. Every solution of the quartic functional equation is said to be a quartic mapping. The stability problem for the quartic functional equation first was solved by Rassias [5] and Lee and Chung [6] for mappings $f: X \rightarrow Y$, where $X$ is a real normed space and $Y$ is a Banach space.

In the sequel, we shall adopt the usual terminology, notations and conventions of the theory of random normed spaces as in [7-15]. Throughout this paper, the space of all probability distribution functions is denoted by

$$
\begin{aligned}
& \Delta^{+}=\{F: \mathbb{R} \cup\{-\infty,+\infty\} \rightarrow[0,1]: F \text { is left-continuous } \\
&\text { and nondecreasing on } \mathbb{R} \text { and } F(0)=0, F(+\infty)=1\}
\end{aligned}
$$

and the subset $D^{+} \subseteq \Delta^{+}$is the set $D^{+}=\left\{F \in \Delta^{+}: l^{-} F(+\infty)=1\right\}$, where $l^{-} f(x)$ denotes the left limit of the function $f$ at the point $x$. The space $\Delta^{+}$is partially ordered by the usual pointwise ordering of functions, that is, $F \leq G$ if and only if $F(t) \leq G(t)$ for all $t$ in $\mathbb{R}$. The maximal element for $\Delta^{+}$in this order is the distribution function given by

$$
\varepsilon_{0}(t)= \begin{cases}0, & \text { if } t \leq 0 \\ 1, & \text { if } t \leq 0\end{cases}
$$

Definition 1.1 (see [13]). A function $T:[0,1] \times[0,1] \rightarrow[0,1]$ is a continuous triangular norm (briefly, a $t$-norm) if $T$ satisfies the following conditions:

(a) $T$ is commutative and associative;

(b) $T$ is continuous;

(c) $T(a, 1)=a$ for all $a \in[0,1]$;

(d) $T(a, b) \leq T(c, d)$ whenever $a \leq c$ and $b \leq d$ for all $a, b, c, d \in[0,1]$.

Three typical examples of continuous $t$-norms are $T(a, b)=a b, T(a, b)=\max (a+b-$ $1,0)$ and $T(a, b)=\min (a, b)$.

Recall that, if $T$ is a $t$-norm and $\left\{a_{n}\right\}$ is a given sequence of numbers in [0,1], $T_{i=1}^{n} a_{i}$ is defined recursively by $T_{i=1}^{1} a_{i}=a_{1}$ and $T_{i=1}^{n} a_{i}=T\left(T_{i=1}^{n-1} a_{i}, a_{n}\right)$ for $n \geq 2$.

Definition 1.2. A random normed space (briefly, $\mathrm{RN}$-space) is a triple $(X, \mu, T)$, where $X$ is a vector space, $T$ is a continuous $t$-norm and $\mu$ is a mapping from $X$ into $D^{+}$such that the following conditions hold:

(PN1) $\mu_{x}(t)=\varepsilon_{0}(t)$ for all $t>0$ if and only if $x=0$;

(PN2) $\mu_{\alpha x}(t)=\mu_{x}(t /|\alpha|)$ for all $x$ in $X, \alpha \neq 0$ and $t \geq 0$;

(PN2) $\mu_{x+y}(t+s) \geq T\left(\mu_{x}(t), \mu_{y}(s)\right)$ for all $x, y \in X$ and $t, s \geq 0$.

Definition 1.3. Let $(X, \mu, T)$ be an $\mathrm{RN}$-space.

(1) A sequence $\left\{x_{n}\right\}$ in $X$ is said to be convergent to $x$ in $X$ if, for every $t>0$ and $\varepsilon>0$, there exists a positive integer $N$ such that $\mu_{x_{n}-x}(t)>1-\varepsilon$ whenever $n \geq N$.

(2) A sequence $\left\{x_{n}\right\}$ in $X$ is called Cauchy sequence if, for every $t>0$ and $\varepsilon>0$, there exists a positive integer $N$ such that $\mu_{x_{n}-x_{m}}(t)>1-\varepsilon$ whenever $n \geq m \geq N$. 
(3) An RN-space $(X, \mu, T)$ is said to be complete if and only if every Cauchy sequence in $X$ is convergent to a point in $X$.

Theorem 1.4 (see [13]). If $(X, \mu, T)$ is an $R N$-space and $\left\{x_{n}\right\}$ is a sequence such that $x_{n} \rightarrow x$, then $\lim _{n \rightarrow \infty} \mu_{x_{n}}(t)=\mu_{x}(t)$.

In this paper, we establish the stability of the cubic and quartic functional equations in the setting of random normed spaces.

\section{On the Stability of Cubic Mappings in RN-Spaces}

Theorem 2.1. Let $X$ be a linear space, $\left(Z, \mu^{\prime}, \min \right)$ be an $R N$-space, $\varphi: X \times X \rightarrow Z$ be a function such that for some $0<\alpha<8$,

$$
\mu_{\varphi(2 x, 0)}^{\prime}(t) \geq \mu_{\alpha \varphi(x, 0)}^{\prime}(t), \quad \forall x \in X, t>0
$$

$f(0)=0$ and $\lim _{n \rightarrow \infty} \mu_{\varphi\left(2^{n} x, 2^{n} y\right)}^{\prime}\left(8^{n} t\right)=1$ for all $x, y \in X$ and $t>0$. Let $(Y, \mu, \min )$ be a complete $R N$-space. If $f: X \rightarrow Y$ is a mapping such that

$$
\mu_{f(2 x+y)+f(2 x-y)-2 f(x+y)-2 f(x-y)-12 f(x)}(t) \geq \mu_{\varphi(x, y)}^{\prime}(t), \quad \forall x \in X, t>0,
$$

then there exists a unique cubic mapping $C: X \rightarrow Y$ such that

$$
\mu_{f(x)-C(x)}(t) \geq \mu_{\varphi(x, 0)}^{\prime}(2(8-\alpha) t)
$$

Proof. Putting $y=0$ in (2.2), we get

$$
\mu_{(f(2 x) / 8)-f(x)}(t) \geq \mu_{\varphi(x, 0)}^{\prime}(16 t), \quad \forall x \in X
$$

Replacing $x$ by $2^{n} x$ in (2.4) and using (2.1), we obtain

$$
\begin{aligned}
\mu_{\left(f\left(2^{n+1} x\right) / 8^{n+1}\right)-\left(f\left(2^{n} x\right) / 8^{n}\right)}(t) & \geq \mu_{\varphi\left(2^{n} x, 0\right)}^{\prime}\left(16 \times 8^{n}\right) \\
& \geq \mu_{\varphi(x, 0)}^{\prime}\left(\frac{16 \times 8^{n}}{\alpha^{n}}\right) .
\end{aligned}
$$

It follows from $\left(f\left(2^{n} x\right) / 8^{n}\right)-f(x)=\sum_{k=0}^{n-1}\left(\left(f\left(2^{k+1} x\right) / 8^{k+1}\right)-\left(f\left(2^{k} x\right) / 8^{k}\right)\right)$ and (2.5) that

$$
\mu_{\left(f\left(2^{n} x\right) / 8^{n}\right)-f(x)}\left(t \sum_{k=0}^{n-1} \frac{\alpha^{k}}{16 \times 8^{k}}\right) \geq T_{k=0}^{n-1}\left(\mu_{\varphi(x, 0)}^{\prime}(t)\right)=\mu_{\varphi(x, 0)}^{\prime}(t)
$$


that is,

$$
\mu_{\left(f\left(2^{n} x\right) / 8^{n}\right)-f(x)}(t) \geq \mu_{\varphi(x, 0)}^{\prime}\left(\frac{t}{\sum_{k=0}^{n-1}\left(\alpha^{k} /\left(16 \times 8^{k}\right)\right)}\right) .
$$

By replacing $x$ with $2^{m} x$ in (2.7), we observe that

$$
\mu_{\left(f\left(2^{n+m} x\right) / 8^{n+m}\right)-\left(f\left(2^{m} x\right) / 8^{m}\right)}(t) \geq \mu_{\varphi(x, 0)}^{\prime}\left(\frac{t}{\sum_{k=m}^{n+m}\left(\alpha^{k} /\left(16 \times 8^{k}\right)\right)}\right) .
$$

As $\mu_{\varphi(x, 0)}^{\prime}\left(t / \sum_{k=m}^{n+m}\left(\alpha^{k} / 16 \times 8^{k}\right)\right)$ tends to 1 as $m, n$ tend to $\infty$, then $\left\{f\left(2^{n} x\right) / 8^{n}\right\}$ is a Cauchy sequence in $(Y, \mu, \mathrm{min})$. Since $(Y, \mu, \mathrm{min})$ is a complete $\mathrm{RN}$-space, this sequence converges to some point $C(x) \in Y$. Fix $x \in X$ and put $m=0$ in (2.8). Then we obtain

$$
\mu_{\left(f\left(2^{n} x\right) / 8^{n}\right)-f(x)}(t) \geq \mu_{\varphi(x, 0)}^{\prime}\left(\frac{t}{\sum_{k=0}^{n-1}\left(\alpha^{k} /\left(16 \times 8^{k}\right)\right)}\right)
$$

and so, for every $\delta>0$, we have

$$
\begin{aligned}
\mu_{C(x)-f(x)}(t+\delta) & \geq T\left(\mu_{C(x)-\left(f\left(2^{n} x\right) / 8^{n}\right)}(\delta), \mu_{\left(f\left(2^{n} x\right) / 8^{n}\right)-f(x)}(t)\right) \\
& \geq T\left(\mu_{C(x)-\left(f\left(2^{n} x\right) / 8^{n}\right)}(\delta), \mu_{\varphi(x, 0)}^{\prime}\left(\frac{t}{\sum_{k=0}^{n-1}\left(\alpha^{k} /\left(16 \times 8^{k}\right)\right)}\right)\right) .
\end{aligned}
$$

Taking the limit as $n \rightarrow \infty$ and using (2.10), we get

$$
\mu_{C(x)-f(x)}(t+\delta) \geq \mu_{\varphi(x, 0)}^{\prime}(2 t(8-\alpha))
$$

Since $\delta$ was arbitrary, by taking $\delta \rightarrow 0$ in (2.11), we get

$$
\mu_{C(x)-f(x)}(t) \geq \mu_{\varphi(x, 0)}^{\prime}(2 t(8-\alpha))
$$

Replacing $x$ and $y$ by $2^{n} x$ and $2^{n} y$ in (2.2), respectively, we get

$$
\begin{aligned}
& \mu_{\left(f\left(2^{n}(2 x+y)\right) / 8^{n}\right)+\left(f\left(2^{n}(2 x-y)\right) / 8^{n}\right)-\left(2 f\left(2^{n}(x+y)\right) / 8^{n}\right)-\left(2 f\left(2^{n}(x-y)\right) / 8^{n}\right)-\left(12 f\left(2^{n}(x)\right) / 8^{n}\right)}(t) \\
& \quad \geq \mu_{\varphi\left(2^{n} x, 2^{n} y\right)}^{\prime}\left(8^{n} t\right)
\end{aligned}
$$

for all $x, y \in X$ and for all $t>0$. Since $\lim _{n \rightarrow \infty} \mu_{\varphi\left(2^{n} x, 2^{n} y\right)}^{\prime}\left(8^{n} t\right)=1$, we conclude that $C$ fulfills (1.1). To prove the uniqueness of the cubic mapping $C$, assume that there exists a 
cubic mapping $D: X \rightarrow Y$ which satisfies (2.3). Fix $x \in X$. Clearly, $C\left(2^{n} x\right)=8^{n} C(x)$ and $D\left(2^{n} x\right)=8^{n} D(x)$ for all $n \in \mathbb{N}$. It follows from (2.3) that

$$
\begin{aligned}
\mu_{C(x)-D(x)}(t) & =\lim _{n \rightarrow \infty} \mu_{\left(C\left(2^{n} x\right) / 8^{n}\right)-\left(D\left(2^{n} x\right) / 8^{n}\right)}(t), \\
\mu_{\left(C\left(2^{n} x\right) / 8^{n}\right)-\left(D\left(2^{n} x\right) / 8^{n}\right)}(t) & \geq \min \left\{\mu_{\left(C\left(2^{n} x\right) / 8^{n}\right)-\left(f\left(2^{n} x\right) / 8^{n}\right)}\left(\frac{t}{2}\right), \mu_{\left(D\left(2^{n} x\right) / 8^{n}\right)-\left(f\left(2^{n} x\right) / 8^{n}\right)}\left(\frac{t}{2}\right)\right\} \\
& \geq \mu_{\varphi\left(2^{n} x, 0\right)}^{\prime}\left(8^{n}(8-\alpha) t\right) \\
& \geq \mu_{\varphi(x, 0)}^{\prime}\left(\frac{8^{n}(8-\alpha) t}{\alpha^{n}}\right) .
\end{aligned}
$$

Since $\lim _{n \rightarrow \infty}\left(8^{n}(8-\alpha) t / \alpha^{n}\right)=\infty$, we get $\lim _{n \rightarrow \infty} \mu_{\varphi(x, 0)}^{\prime}\left(8^{n}(8-\alpha) t / \alpha^{n}\right)=1$. Therefore, it follows that $\mu_{C(x)-D(x)}(t)=1$ for all $t>0$ and so $C(x)=D(x)$. This completes the proof.

\section{On the Stability of Quartic Mappings in RN-Spaces}

Theorem 3.1. Let $X$ be a linear space, $\left(Z, \mu^{\prime}, \min \right)$ be an $R N$-space, $\varphi: X \times X \rightarrow Z$ be a function such that for some $0<\alpha<16$,

$$
\mu_{\varphi(2 x, 0)}^{\prime}(t) \geq \mu_{\alpha \varphi(x, 0)}^{\prime}(t), \quad \forall x \in X, t>0,
$$

$f(0)=0$ and $\lim _{n \rightarrow \infty} \mu_{\varphi\left(2^{n} x, 2^{n} y\right)}^{\prime}\left(16^{n} t\right)=1$ for all $x, y \in X$ and $t>0$. Let $(Y, \mu, \min )$ be a complete $R N$-space. If $f: X \rightarrow Y$ is a mapping such that

$$
\mu_{f(2 x+y)+f(2 x-y)-4 f(x+y)-4 f(x-y)-24 f(x)+6 f(y)}(t) \geq \mu_{\varphi(x, y)}^{\prime}(t), \quad \forall x \in X, t>0,
$$

then there exists a unique quartic mapping $Q: X \rightarrow Y$ such that

$$
\mu_{f(x)-Q(x)}(t) \geq \mu_{\varphi(x, 0)}^{\prime}(2(16-\alpha) t) .
$$

Proof. The proof is the same as Theorem 2.1.

\section{Acknowledgments}

The authors would like to thank the area editor Prof. Wing-Sum Cheung and two anonymous referees for their valuable comments and suggestions. The third author was supported by the Korea Research Foundation Grant funded by the Korean Government (KRF-2008-313C00050). 


\section{References}

[1] E. Baktash, Y. J. Cho, M. Jalili, R. Saadati, and S. M. Vaezpour, "On the stability of cubic mappings and quadratic mappings in random normed spaces," Journal of Inequalities and Applications, vol. 2008, Article ID 902187, 11 pages, 2008.

[2] K.-W. Jun and H.-M. Kim, "The generalized Hyers-Ulam-Rassias stability of a cubic functional equation," Journal of Mathematical Analysis and Applications, vol. 274, no. 2, pp. 867-878, 2002.

[3] Y.-S. Lee, "Stability of a quadratic functional equation in the spaces of generalized functions," Journal of Inequalities and Applications, vol. 2008, Article ID 210615, 12 pages, 2008.

[4] K.-W. Jun, H.-M. Kim, and I.-S. Chang, "On the Hyers-Ulam stability of an Euler-Lagrange type cubic functional equation," Journal of Computational Analysis and Applications, vol. 7, no. 1, pp. 21-33, 2005.

[5] J. M. Rassias, "Solution of the Ulam stability problem for quartic mappings," Glasnik Matematički, vol. 34, no. 2, pp. 243-252, 1999.

[6] Y.-S. Lee and S.-Y. Chung, "Stability of cubic functional equation in the spaces of generalized functions," Journal of Inequalities and Applications, vol. 2007, Article ID 79893, 13 pages, 2007.

[7] S. Chang, Y. J. Cho, and S. M. Kang, Nonlinear Operator Theory in Probabilistic Metric Spaces, Nova Science, Huntington, NY, USA, 2001.

[8] O. Hadžić and E. Pap, Fixed Point Theory in Probabilistic Metric Spaces, vol. 536 of Mathematics and Its Applications, Kluwer Academic Publishers, Dordrecht, The Netherlands, 2001.

[9] D. Miheț and V. Radu, "On the stability of the additive Cauchy functional equation in random normed spaces," Journal of Mathematical Analysis and Applications, vol. 343, no. 1, pp. 567-572, 2008.

[10] D. Miheț, R. Saadati, and S. M. Vaezpour, "The stability of the quartic functional equation in random normed spaces," Acta Applicandae Mathematicae, 2009.

[11] D. Miheț, R. Saadati, and S. M. Vaezpour, "The stability of an additive functional equation in Menger probabilistic $\varphi$-normed spaces," Mathematica Slovaca. In press.

[12] R. Saadati and S. M. Vaezpour, "Linear operators in finite dimensional probabilistic normed spaces," Journal of Mathematical Analysis and Applications, vol. 346, no. 2, pp. 446-450, 2008.

[13] B. Schweizer and A. Sklar, Probabilistic Metric Spaces, North-Holland Series in Probability and Applied Mathematics, North-Holland, New York, NY, USA, 1983.

[14] A. N. Šerstnev, "On the concept of a stochastic normalized space," Doklady Akademii Nauk SSSR, vol. 149 , pp. 280-283, 1963 (Russian).

[15] S. Shakeri, "Intuitionistic fuzzy stability of Jensen type mapping," The Journal of Nonlinear Sciences and Its Applications, vol. 2, pp. 105-112, 2009. 